\title{
HLA-A24: FACTOR DE RIESGO EN RETINOPATÍA DIABÉTICA PROLIFERANTE
}

\section{HLA-A24: RISK FACTOR IN PROLIFERANTE DIABETIC RETINOPATHY}

\author{
ASENSIO-SÁNCHEZ VM ${ }^{1}$, RODRÍGUEZ-DELGADO B ${ }^{2}$, GARCÍA-HERRERO E², \\ CABO-VAQUERA V ${ }^{2}$, GARCÍA-LOYGORRI C ${ }^{1}$
}

\section{RESUMEN}

Objetivo: La retinopatía diabética proliferante (RDP) se caracteriza por pérdida de la visión en la población joven. Está bien establecido que el antígeno leucocitario humano (HLA)-A24 es un factor de riesgo para la pérdida total de las células, del páncreas. Nuestro objetivo es estudiar la asociación del HLA-A24 con la RDP.

Material y método: Se estudió un grupo de pacientes con RDP $(n=95)$ y un grupo control $(n=60)$. A todos se les determinó el HLA-A24 mediante técnicas hibridación molecular.

Resultados: El grupo control mostró menos frecuencia de HLA-A24 que el grupo con RDP ( $\mathrm{p}=$ $0,043)$. El HLA-A24 se asoció a retinopatía diabética proliferante $(\mathrm{OR}=5,4 ; 95 \% \mathrm{CI}=3,2-7,6 ; \mathrm{p}<$ 0,001 ).

Conclusiones: El HLA-A24 no es un factor de protección para la retinopatía diabética proliferante, es un factor de riesgo para desarrollarla.

Palabras clave: Diabetes insulindependiente, retinopatía diabética proliferante, HLA, HLA-A24, factor de riesgo.

\begin{abstract}
Objective: Proliferative diabetic retinopathy (PDR) is characterized by a progressive visual impairment in young people. Human leucocyte antigen (HLA)A24 is a well-established factor associated with the pancreatic islets of Langerhans lost in this process. Our aim was to study further the relationship of the HLA-A24 associated with PDR.

Materials and methods: We evaluated a group of patients with PDR ( $\mathrm{n}=95)$ and a healthy control group $(n=60)$. HLA-A24 for each participant in the study was determined by molecular hybridization techniques.

Results: The control group showed a lower frequency of HLA-A24 compared with the PDR group $(\mathrm{p}=0.043)$. HLA-A24 was associated with PDR $(\mathrm{OR}=5.4 ; 95 \% \mathrm{CI}=3.2-7.6 ; \mathrm{p}<0.001)$.

Conclusions: HLA-A24 is not a protective factor for PDR, but is a risk factor of its development (Arch Soc Esp Oftalmol 2007; 82: 753-756).

Key words: Insulin-dependent diabetes, proliferative diabetic retinopathy, HLA, HLA-A24, risk factor.
\end{abstract}

\footnotetext{
Recibido: 26/11/06. Aceptado: 30/10/07.

Hospital General. Medina del Campo. Valladolid. España.

1 Doctor en Medicina.

2 Licenciado en Medicina.

Correspondencia:

V.M. Asensio Sánchez

Hospital General Servicio Castellano-Leonés de Salud

Servicio de Oftalmología

Medina del Campo (Valladolid)

España

E-mail: vasensio@hmdc.sacyl.es
} 


\section{INTRODUCCIÓN}

El tiempo de evolución de la diabetes y la hiperglucemia crónica son los factores de riesgo más conocidos y establecidos para el desarrollo de retinopatía diabética, pero existe un grupo de pacientes que a pesar de un mal control glucémico no desarrollan en su evolución retinopatía diabética y otros que con un control riguroso y estricto desarrollan formas severas de retinopatía incluso resistentes a diferentes terapias $(1,2)$. El sistema antigénico leucocitario humano (HLA) tiene importancia en la respuesta y tolerancia del sistema inmunitario y en pacientes con diabetes tipo 1 se ha relacionado la retinopatía con antígenos en la región DQ (3). Se ha descrito $(4,5)$ una fuerte relación entre la presencia de HLA-A24 y la completa destrucción de las células beta de los islotes pancreáticos en los diabéticos insulino-dependientes, por lo que se puede especular que en estos pacientes la retinopatía se desarrollará antes y será más agresiva que en los pacientes diabéticos insulino-dependientes HLA-A24 negativo. Partiendo de esta premisa se planificó el estudio que se presenta, siendo su objetivo establecer la relación entre HLA-A24 y la retinopatía diabética en sus formas más severas en una muestra de pacientes.

\section{SUJETOS, MATERIAL Y MÉTODOS}

Este estudio fue aprobado por la Comisión de Investigación y Docencia de nuestro hospital. Los pacientes se reclutaron de la consulta de retina y de la consulta general. Se estudiaron 95 pacientes diabéticos insulino-dependientes entre los años 1998 y 2005. Los criterios de inclusión para los pacientes fueron los siguientes:

- Aceptar participar en el estudio con firma del consentimiento informado.

- Diabetes mellitus insulino-dependiente tratada por endocrino, internista o médico de atención primaria, con diez o más años de evolución.

- Diagnóstico de retinopatía diabética no proliferante severa o retinopatía diabética proliferante (RDP).

- Poder acceder a su historial clínico.

En todos los pacientes fue realizado un estudio de fondo de ojo bajo midriasis por un mismo oftalmólogo y fueron clasificados para este estudio como retinopatía diabética no proliferante severa/ retino- patía proliferante $(\mathrm{RDP})(\mathrm{n}=95)$. El grupo control fueron 60 familiares sanos.

En cada caso se realizó un tipaje HLA-A24 por hibridación molecular en sangre total (PCR/SSO).

Se calculó la asociación entre el HLA-A24 y RDP mediante el cálculo de la odds ratio (OR) y su correspondiente intervalo de confianza a un grado de confianza del $95 \%$. El análisis por sexo y edad se realizó a través de una regresión logística.

\section{RESULTADOS}

En la tabla I se muestran las características de la población estudiada no existiendo diferencias estadísticas entre los casos y controles. En la tabla II se presenta el grado de significación del HLA-A24 en los pacientes frente al grupo control y el resultado del cálculo de asociación (OR) de este HLA con el grupo de pacientes respecto al grupo control. El riesgo dependiente de HLA-A24, indica que la probabilidad de padecer RDP se incrementa en un factor de 5 veces (IC 95\%=3,4-8,3; $<<0,001$ ). El grupo control muestra menos frecuencia de HLA-A24 (11\%) comparado con el grupo de pacientes $(55 \%)$ $(\mathrm{p}=0,043)$. La asociación de HLA-A24 es significativa en todos los grupos de edad: 30-40 años: $\mathrm{OR}=11,2$; IC 95\%= 4,76-24,80; 40-50 años: OR= 7,26; IC 95\%= 3,4-19,80; 50-60 años: OR= 10,2; IC $95 \%=4,87-21,70 ; 60-70$ años: $O R=11,6 ;$ IC $95 \%=4,66-23,40 ; 70-80$ años: OR= 7,23; IC 95\%=

Tabla I. Características demográficas

\begin{tabular}{lcc}
\hline & RDP & Control \\
\hline N. & 95 & 60 \\
Sexo & $63,16 \%$ mujeres & $66,67 \%$ mujeres \\
Edad: & 66,5 D.E. 5,8 & 63,3 D.E. 4,2 \\
Edad de diagnóstico: & $43,9 \pm 7,6$ años & \\
\hline \hline
\end{tabular}

RDP: retinopatía diabética proliferante/retinopatía diabética no proliferante severa; N..$^{\circ}$ número.

Tabla II. HLA-A24 y grado de asociación con RDP frente a los controles

\begin{tabular}{lccc}
\hline & \% de HLA-A24 & OR & IC \\
\hline RDP & $55 \%$ & 5,4 & $3,2-7,6(\mathrm{p}<0,001)$ \\
Control & $11 \%$ & & \\
\hline \hline
\end{tabular}

HLA: Antígeno leucocitario humano; OR: odds ratio; IC: intervalo de confianza; RDP: Retinopatía diabética proliferante/ no proliferante severa. 
3,45-18,76. HLA-A24 se asocia a RDP de forma similar en hombres $(\mathrm{OR}=5,1 ; \mathrm{IC} 95 \%=2,57-10,21)$ $\mathrm{y}$ en mujeres $(\mathrm{OR}=5,31$; IC $95 \%=2,87-11,34)$.

\section{DISCUSIÓN}

Está claramente establecido que el control estricto de la glucemia es la mejor herramienta que tiene el clínico para la prevención de la retinopatía diabética (1), no obstante existe un grupo de pacientes diabéticos que desarrollarán retinopatía diabética a pesar de un buen control y otros no la desarrollarán a pesar de un control pobre, esto puede indicar que los factores de riesgo para diabetes y retinopatía no tienen por que ser los mismos y que factores genéticos pueden influir en la susceptibilidad de desarrollar retinopatía severa (2).

La diabetes tipo 1 es causada por un fallo en el sistema autoinmune y se asocia a determinados HLA (3). El HLA es sumamente interesante por las numerosas implicaciones que tiene en biología y medicina, pero quizá lo más llamativo sea la influencia que este sistema antigénico ejerce en la susceptibilidad a gran número de enfermedades (6).

En el desarrollo de la retinopatía diabética la influencia de los factores genéticos han sido estudiados con menos interés por ser un tema que hasta hace poco era considerado alejado de la oftalmología y porque los estudios HLA son caros y los laboratorios no tienen montada la técnica de los tipos menos frecuentes como es el caso del HLA-A24. En los diabéticos insulino-dependientes el número de islotes residuales de células, va a influenciar el mejor o peor control glucémico y la completa destrucción de los islotes de Langerhans se relaciona con la presencia de HLA-A24 $(4,5)$.

Hay trabajos que hacen referencia a la influencia del HLA como marcador genético de la retinopatía diabética $(7,8)$ considerando al sistema HLA como el mayor factor de riesgo para el desarrollo de retinopatía diabética proliferante, independientemente de los niveles glucémicos.

En este trabajo se ha pretendido estudiar la relación entre el HLA-A24 y la RDP, en un grupo de pacientes frente a un grupo control. El grupo control al estar formado por familiares de pacientes con RDP, minimiza el efecto de las variables medioambientales y económico-sociales que pueden influir en el desarrollo de la diabetes. De ahí que sea recomendable estudiar grupos lo más homogéneos posi- bles. En nuestra población se confirma la relación entre el HLA-A24 y la RDP y su presencia aumenta en cinco veces el riesgo de padecerla. El HLAA24 tiene una relación significativa con la edad en todos los grupos y es independiente del sexo. La asociación entre el HLA-A24 y la total destrucción de los islotes pancreáticos $(4,5)$ significa una carencia total de insulina con un control glucémico malo durante las 24 horas del día, presentando hiperglucemias exageradas seguidas de hipoglucemias intensas lo que produce una pérdida total de la autorregulación vascular.

Los pacientes de Nakanishi K et al. (10) con completa destrucción de las células , progresaron a formas severas de retinopatía diabética precozmente y el $89,6 \%$ de los pacientes tenían HLA-A24. Honeyman MC et al. (11) en un análisis con familias diabéticas insulinodependientes consideran que el HLA predispone e influencia la progresión de la diabetes. Mimura T et al. (12) establecen que varios tipos de HLA I y HLA II indican el pronóstico de la retinopatía en pacientes con diabetes tipo 1 . En conclusión, nuestro trabajo muestra que el HLA-A24 es un factor de riesgo para desarrollar las formas más severas de retinopatía diabética, a pesar de esto, no recomendamos el estudio sistemático de HLA-A24 como un test de diagnóstico, pero consideramos que es importante para establecer grupos de intervención con medidas de prevención desde el inicio.

\section{BIBLIOGRAFÍA}

1. Ferris FL, Davis MD, Aiello LM. Treatment of diabetic retinopathy. N Engl J Med 1999; 341: 667-678.

2. Mimura T, Amano S, Kato S, Araie M, Funatsu H, Kitano $S$, et al. HLA typing is not predictive of proliferative diabetic retinopathy in patients with younger onset type 2 diabetes mellitus. Br J Ophthalmol 2004; 88: 303-305.

3. Agardh D, Gaur LK, Agardh E, Landin-Olsson M, Agardh $C D$, Lernmark A. HLA-DQB1*0201/0302 is associated with severe retinopathy in patients with IDDM. Diabetologia 1996; 39: 1313-1317.

4. Nakanishi $K$, Kobayashi $T$, Murase T, Nakatsuji $T$, Inoko $H$, Tsuji K, et al. Association of HLA-A24 with complete beta-cell destruction in IDDM. Diabetes 1993; 42: 10861093.

5. Nakanishi $K$, Kobayashi T, Murase T, Naruse T, Nose $Y$, Inoko H. Human leukocyte antigen-A24 and-DQA1*0301 in Japanese insulin-dependent diabetes mellitus: independent contributions to susceptibility to the disease and additive contributions to acceleration of beta-cell destruction. J Clin Endocrinol Metab 1999; 84: 3721-3725. 
6. Diego Acosta M. Sistema HLA: Estructura, genética y función. Tiempos médicos 1985; 303: 10-26.

7. Stewart LL, Field LL, Ross S, McArthur RG. Genetic risk factors in diabetic retinopathy. Diabetologia 1993; 36: 1293-1298.

8. Barbosa J, Ramsay RC, Knobloch WH, Cantrill HL, Noreen $H$, King $R$, et al. Histocompatibility antigen frequencies in diabetic retinopathy. Am J Ophthalmol 1980; 90: 148153.

9. Cruickshanks KJ, Vadheim CM, Moss SE, Roth MP, Riley WJ, Maclaren NK, et al. Genetic marker associations with proliferative retinopathy in persons diagnosed with diabetes before 30 yr of age. Diabetes 1992; 41: 879-889.
10. Nakanishi K, Kobayashi T, Inoko H, Tsuji K, Murase T, Kosaka K. Residual beta-cell function and HLA-A24 in IDDM. Markers of glycemic control and subsequent development of diabetic retinopathy. Diabetes 1995; 44: 1334-1339.

11. Honeyman MC, Harrison LC, Drummond B, Colman PG, Tait BD. Analysis of families at risk for insulin-dependent diabetes mellitus reveals that HLA antigens influence progression to clinical disease. Mol Med 1995; 5: 576-582.

12. Mimura T, Funatsu H, Uchigata Y, Kitano S, Noma H, Shimizu E, et al. Relationship between human leukocyte antigen status and proliferative diabetic retinopathy in patients with younger-onset type 1 diabetes mellitus. Am J Ophthalmol 2003; 135: 844-848. 